\title{
Chapter 10 \\ Technical Aspects of Biosecurity: Screening \\ Guidance, Attribution, and Traceability
}

\author{
Evan Appleton and Piers Millett
}

\subsection{Introduction}

Biosecurity is a multi-disciplinary topic that covers areas of policy, public health, economics, and science. This chapter focuses on the technical scientific aspects of the current international biosecurity framework. We discuss these technical areas in terms time horizon. We begin this chapter with review of current technology within the international biosecurity framework and discuss weakness and opportunities for further work. We then focus on near-term technical developments and imminent opportunities to strengthen the existing framework. Specifically, we break down the range of issues into biological threat prevention, detection, and response. We discuss how technical tools can assist in policy development and the engineering cycle of Design, Built, and Test. Finally, this chapter describes a 'futuring' exercise conducted by the working group that created this chapter to explore broader longerterm issues in the biosecurity space.

\footnotetext{
E. Appleton ( $\bowtie)$

Department of Genetics, Harvard Medical School, Boston, MA, USA

Wyss Institute for Biologically Inspired Engineering at Harvard University,

Boston, MA, USA

e-mail: evan_appleton@hms.hardvard.edu

P. Millett

iGEM Foundation, Boston, MA, USA
}

Future of Humanity Institute, University of Oxford, Oxford, UK

(C) The Author(s) 2021

B. D. Trump et al. (eds.), Emerging Threats of Synthetic Biology and Biotechnology, NATO Science for Peace and Security Series C:

Environmental Security, https://doi.org/10.1007/978-94-024-2086-9_10 


\subsection{Current Technology}

Much recent work within our current biosecurity architecture focuses on tracking of DNA sequences that are considered to be (or may be) hazardous. This framework can be further separated into two general categories: (1) Screening for hazardous DNA sequences prior to DNA synthesis and (2) DNA sequencing and repository screening for attribution of the source of physical DNA that already exists. This framework has been developed gradually over the past ten plus years by multiple organizations. It includes policies and technical tools implemented from stakeholders across industry, academia, and government.

While this collection of approaches has been developed by multiple parties to provide coverage of the broader biosecurity area, it currently exists as a fragmented framework that has a number of clear gaps, some of which are currently being addressed by a number of parties. These gaps include but are not limited to: laborious assessment of false-positive hits from security screens, no standard guideline for implementation of a universal tool (or set of tools), and no well-defined database of established threats or potential threats.

\subsubsection{DNA Sequence Screening}

\subsubsection{State-of-the-Art}

Companies across the world have been offering DNA synthesis as a service for recombinant DNA biotechnology for the past several decades. This technology has improved exponentially in recent years, and currently exists at a scale where many companies are able to produce custom DNA sequences for customers on the near mega-base scale. While this capability has dramatically enabled biotechnology to make a litany of breakthroughs across biology, it has also introduced a key question to the scientific community: are there DNA sequences that DNA synthesis providers should not provide to certain customers? And furthermore - are there some sequences that should not be generated and provided to any customers?

The general answer to both of these questions by the scientific community is "yes". This answer, however, is highly conditional based on a variety of factors. While the vast majority of scientists request DNA sequences that pose no known threat, sometimes sequences of known (or potential hazard) are requested for DNA synthesis. In this situation, there is a general consensus that there are some sequences that should only be provided to qualified customers and some sequences that should not be provided to anyone. Currently, a majority of large DNA synthesis providers screen orders for the presence of viral, toxic, or otherwise known hazardous sequences to avoid sending hazardous DNA to an unqualified end user. This process typically involves lookups using local alignment tools against a reference database of hazardous sequences. 
In 2009, five DNA synthesis providers founded a consortium to create guidelines for screening synthetic DNA orders, called the International Gene Synthesis Consortium (IGSC). ${ }^{1}$ IGSC provides its members guidelines for implementing synthesis screening and gives its members benchmark tests to assure that their tools meet the current screening standards. The consortium also meets regularly to discuss any needed updates to existing protocols. It is reported that approximately $80 \%$ of global DNA synthesis is provided by currently IGSC members performing synthesis screening. However, since IGSC itself does not manage a consensus software tool or database for all members and membership to IGSC is voluntary (and not a legal mandate), there is no unified implementation of these protocols and a lot of the nuanced decision making on whether or not to synthesize a sequence is still left to individual parties. No detailed guidance exists on how to handle specific edge cases where the threat potential of a sequence can't be determined.

In parallel with IGSC activities, the US HHS published a screening framework guidance ${ }^{2}$ for all synthesis providers to use when developing their screening tools. This guidance was made with recommendations in mind from a US National Research Council report on the feasibility of identifying DNA sequences of concern. ${ }^{3}$ This guidance has been put to use by some non-IGSC DNA synthesis providers, ${ }^{4}$ but as with IGSC member tools, the details of how the screening is done specifically is left to the individual parties. Other large synthetic biology groups like the International Genetically Engineered Machine (iGEM) competition and Engineering Biology Research Consortium (EBRC) have their own biosecurity considerations that sometimes employ IGSC member tools, but do not currently widely use independently built DNA screening tools or have not published their own screening guidelines.

\subsubsection{Next-Generation Tool Development}

More recently, a number of other coordinated research efforts have been initiated to fill existing gaps in the global sequence-screening framework. These have included technical research programs, screening guidance updates, and attempts to develop an open-source tool for general use across the world.

In 2018, the US Intelligence Advanced Research Projects Activities (IARPA) program announced the creation of two research programs to build new tools to identify DNA sequences that pose a safety hazard or have been engineered ${ }^{5,6}$ The

\footnotetext{
${ }^{1}$ https://genesynthesisconsortium.org/

${ }^{2}$ https://www.phe.gov/Preparedness/legal/guidance/syndna/Documents/syndna-guidance.pdf

${ }^{3}$ National Research Council. Sequence-based classification of select agents: a brighter line. National Academies Press, 2010.

${ }^{4}$ https://bliss.jgi.doe.gov/

${ }^{5}$ https://www.iarpa.gov/index.php/research-programs/fun-gcat

${ }^{6} \mathrm{https}: / / w w w . i a r p a . g o v / i n d e x . p h p / r e s e a r c h-p r o g r a m s / f e l i x$
} 
former, called Fun GCAT (Functional Genetic Categorization of Threats), aims to "to develop new approaches and tools for the screening of nucleic acid sequences, and for the functional annotation and characterization of genes of concern, with the goal of preventing the accidental or intentional creation of a biological threat." This program includes development of both new computational and experimental technologies to identify known or novel DNA threat sequences. The latter program, called FELIX (Finding Engineering-Linked Indicators), aims to "to develop new experimental and computational tools to detect engineered biological systems". Together with the technologies developed under the Fun GCAT program, this research initiative has spurned the development of new tools to identify existing and novel threats more efficiently and at a higher standard than the first wave of tools currently implemented in government, academia, and industry.

In parallel with the efforts to build better technologies for sequence screening, the IGSC issued updated guidance to DNA synthesis providers based on new information since 2010. ${ }^{7}$ This updated guidance has provided synthesis providers with additional recommendations on how to perform their sequence screening with newer data resources and recommendations on customer screening.

Finally, there have been recent efforts to use the most up-to-date guidance and resources to develop a universal DNA screening tool that could be used by and DNA synthesizer across the world. This effort has been spearheaded by the Nuclear Threat Initiative (NTI-bio), ${ }^{8}$ in partnership with the World Economic Forum (WEF) and the NTI Biosecurity Innovation and Risk Reduction Initiative. This group has established a working group to consider the technical requirements and implementation options for an international, common screening mechanism to prevent illicit gene synthesis. The group seeks to develop, update, and expand geographic buy-in for screening and convenes specialists from gene synthesis companies, experts from the synthetic biology community, and biosecurity professionals. The mechanism will consider DNA synthesis screening options for commercial providers, as well as producers of desktop synthesizers in the future. Central to the work of this group will be recommendations for making the mechanism common, globally accessible, and regularly updated based on advances in scientific understanding. NTI and WEF have subsequently released these recommendations for consideration during the January 2020 World Economic Forum Annual Meeting in Davos, Switzerland. ${ }^{9}$

\footnotetext{
${ }^{7}$ https://genesynthesisconsortium.org/wp-content/uploads/IGSCHarmonizedProtocol11-21-17.pdf ${ }^{8} \mathrm{https}: / /$ www.nti.org/about/biosecurity/

${ }^{9} \mathrm{https} / / /$ media.nti.org/documents/Biosecurity_Innovation_and_Risk_Reduction.pdf
} 


\subsubsection{Attribution and Traceability}

Screening DNA sequences prior to synthesis is the first technical protective measure used to assure that potentially hazardous sequences are not manufactured for unqualified end-users. But, another set of concerns is how to track and attribute DNA materials that already have already been produced, either by DNA synthesis or DNA assembly. We refer to this problem as the problem of attribution and traceability - specifically, can we determine which organization an engineered piece of DNA came from and what its engineering history might have been. While this area is currently less developed than screening prior to synthesis, there are already some tools and guidelines that have been established for this area.

In the past couple years, at least a couple have tools have been published with identifying the lab-of-origin of engineered DNA. ${ }^{10,11}$ There has also been a competition to test and improve these tools. ${ }^{12}$ The general methodologies use machinelearning methods on large databases of DNA sequences to detect patterns of DNA that can be associated with certain research groups. This tends to be useful because DNA is often shared within labs and varies considerably across laboratories or even small groups within the same lab.

One difficult aspect in the attribution and traceability area is a non-perfect match between digital DNA information and physical DNA. Given that many software tools have been quickly developed in recent years with high variability in logging and tracking standards, there are perhaps many records of physical DNA in databases that might not actually physically exist anywhere. A key example of this problem is the iGEM Registry - it is deliberately open-source to allow people from all over the world to enter DNA sequence information, but many of these sequences were never actually physically built. Furthermore, in many cases, the history and real physical location of these DNA fragments might be unknown. This throws a hitch in the efforts for attribution, as it cannot be taken for granted that the data they operate on maps to real physical DNA sequences in a lab.

In parallel with tools to for attribution and tracing, updates to guidelines for synthesis providers have included considerations for customer screening. Namely, the idea is that synthesis providers should not be sending DNA materials to unqualified users or institutions. For example, in 2006, a journalist at The Guardian was able to obtain a fragment of the Smallpox virus (Variola major) via having the DNA sent to their apartment. ${ }^{13}$ Since this initial oversight by some synthesis providers, it has been the policy of most synthesis providers to verify that DNA is only shipped to

\footnotetext{
${ }^{10}$ Nielsen, Alec AK, and Christopher A. Voigt. "Deep learning to predict the lab-of-origin of engineered DNA." Nature communications 9.1 (2018): 1-10.

${ }^{11}$ Wang, Qi, et al. "PlasmidHawk: Alignment-based Lab-of-Origin Prediction of Synthetic Plasmids." BioRxiv (2020).

${ }^{12} \mathrm{https} / / /$ altlabs.tech/geac/

${ }^{13} \mathrm{https}: / /$ www.theguardian.com/world/2006/jun/14/terrorism.topstories3\#: :text=DNA\%20 sequences $\% 20$ from $\% 20$ some $\% 20$ of,short $\% 20$ sequence $\% 20$ of $\% 20$ smallpox $\% 20$ DNA.
} 
qualified research institutions, but this is only the first step of the problem - the problem is further compounded by the fact that some researchers actively study infectious agents and develop vaccines. In this case, these scientists have a valid reason to use synthetic DNA in their studies, but this must be determined on a caseby-case basis. One additional factor that complicates issues even further in some cases is that it isn't necessarily clear what links any one institution's or researcher's are between academia, industry, or government. Between all of these factors, synthesis providers often result to making complex judgment calls about whether or not to synthesize certain DNA for a particular customer and must invest internal resources to make these decisions.

Another complex issue on the horizon in this space is the development of benchtop DNA synthesizers. These devices would allow operators to synthesize DNA in house, reducing the need to order from a provider likely to screen an order. The details of how to manage biosecurity in this case are currently being heavily debated. While the manufacturers of these machines will add software to control for illicit DNA synthesis in some way, it is unclear what methods will be completely effective. In response, some government have implemented export controls on "Nucleic acid assemblers and synthesizers, which are partly or entirely automated, and designed to generate continuous nucleic acids greater than 1.5 kilobases in length with error rates less than $5 \%$ in a single run". ${ }^{14}$ This problem is still in the early days and the solution is far from robust.

In spite of the complexities of attribution and tracing of DNA synthesis, there is some reason for optimism in this space - those who are interested in pursuing illicit DNA synthesis have historically resorted to the methods easily detectable by simple biosecurity measures. Since biology is still done largely by hand as opposed to fully automated systems, most biologists are not even aware of their habits and will probably naïvely make easy-to-catch mistakes if they decide to pursue illicit DNA synthesis. A naïve bad actor is unlikely to reinvent the wheel, and rather, simply order near full-length pathogen sequences that will get flagged in existing frameworks. A sophisticated bad actor would be very hard to catch under current standards, but most security frameworks in other domains (i.e. cyber-security) are geared towards catching naïve bad actors. While it is important for the community to quickly develop more robust policies and tools for attribution and control of materials, there so far haven't been many examples of pressing issues of the existing framework.

\footnotetext{
${ }^{14}$ Australia Group. "Control List of Dual-use Biological Equipment and Related Technology and Software." (2020) https://www.dfat.gov.au/publications/minisite/theaustraliagroupnet/site/en/ dual_biological.html
} 


\subsubsection{Gaps in Existing Biosecurity Technology Framework}

While we have described the formation of a technical biosecurity framework that has partial solutions for some of the problems identified in the DNA biosecurity space, there are some key shortcomings in the existing framework. Some of the key outstanding issues include:

- High false positive rate - false alarm rates in the approximate range of $\sim 2 \%$ of screened orders are observed and must be examined by hand

- Pathogenic genes or gene fragments embedded in non-pathogenic host sequences will have low match scores and will be missed

- Matching is slow and requires significant computational power since each order must be matched to each reference pathogen

- Black list databases of pathogens may be dangerous to share with the public since they provide bad actors with a concise like of sequences of concern

- Human genes used to interfere with the metabolism will not be captured by the current guideline (e.g. the insulin gene expressed under a strong promoter)

These problems are generally acknowledged by the biosecurity community and are often debated in ongoing meetings and conferences. Each synthesis provider currently addresses these gaps in a different capacity, and some providers are not able to address some of these gaps at all for various reasons. Furthermore, there exist some more nebulous gaps that will need to be addressed at some point, even though they are not currently pressing issues. Some of these include:

- Dissemination of viral and microbial sequences that are not currently on select agents lists, but cannot be ruled out as not dangerous in a general sense

- The use of synthetic biology in virology and gene therapy fields (generally for vaccine development)

- Economic drivers running synthetic biology might be contrary to biosecurity

- Lack of consensus and standards on which parties should be screening DNA synthesis orders

- Widespread use of bench-top synthesis screening technologies fundamentally places a majority of the responsibility of using best biosecurity practices in the customer's hands as opposed to larger institutions

- The growth of DIY synthetic biology and community labs

While there are many active conversations across the community about addressing these problems, there is no existing consensus agreement on these issues. Fundamentally, many of these issues boil down to that we do not yet have good generalizable ways to measure biological threats with clear, meticulous scientific methods. While standardization like the ISO standards ${ }^{15}$ suggested would be nice, at the moment it is too hard to really implement.

\footnotetext{
${ }^{15}$ https://www.iso.org/standards.html
} 
Generally speaking, biosecurity can't be taken for granted - it comes with costs that have to be built in, regardless of the provider. Funders or investors of synthesis technologies must support this and it is often not in today's world. It has been suggested that another route for incentivizing this technology development could be done via paying for liability protection and a regulatory framework to enforce fines and financial consequences of bad practices that result in illicit DNA synthesis. However, this idea is still far off from any form of implementation.

\subsection{Imminent Opportunities for Technology Development}

Thus far, we have outlined existing technical aspects of biosecurity framework and identified some gaps. We identified a set of imminent opportunities for additional technology development that would be beneficial in the short-term. We also noted that other gaps are likely to only be possible to address in the medium- to long-term future We break these opportunities into biological threat Prevention, Detection, and Response as general categories. We further disseminate these areas into more specific topics in each section (Fig. 10.1).

\subsubsection{Biological Threat Prevention}

Since biological engineering projects are now often done through cycles of Design, Building, and Testing, we discuss these imminent threat prevention opportunities below in that context. We identify the current approaches in these areas and suggest key ways in which new technology could be developed in these areas to strengthen biosecurity.

\section{Prevent}

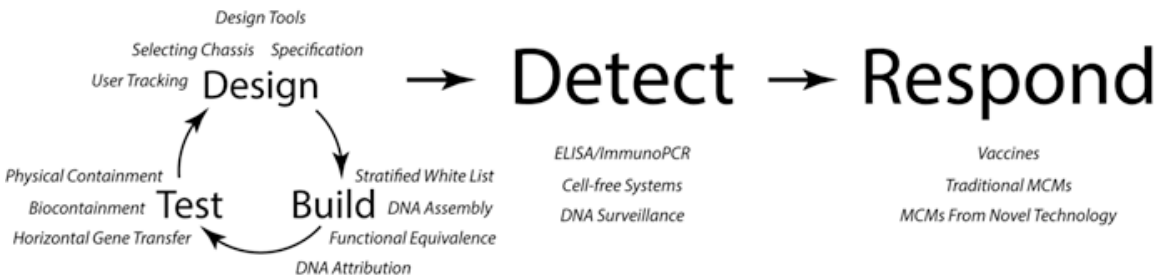

Fig. 10.1 Overview of areas for technology development in current biosecurity landscape. There is a need for technology development in all parts of the biosecurity workflow, starting from the prevention of future threat and ending with responding to bio-threats 


\subsubsection{Design}

The first part of the engineering process is the Design phase. This is when the biological engineer makes key decisions about what DNA sequences will be involved in a project and which function each DNA element is supposed to have. In this starting step lies an opportunity to incorporate biosecurity features of synthetic DNA from the very beginning. In recent years, a number of software tools have been created for synthetic biology that automate the design process, ${ }^{16}$ so there exists an opportunity to add tools specific to biosecurity in these frameworks.

\section{Specification}

The first step of a design process is an abstract design process, where the high-level design requirements are defined before a concrete design with specific real components are formulated. This step is called 'Specification'. Some examples of a specification that have been used in synthetic biology include logic gate behaviour, toggle switch behaviour, and oscillatory behaviour - for these examples, the very high level intentions of a genetic construct are defined, but no actual DNA sequences are yet selected. This step is useful because it defines the overall purpose of an engineered system before committing to the actual components needed to create a fully designed system. This specification is then generally fed automatically into a downstream design tool that chooses and arranges components to satisfy the specification.

While some software tools for specification in synthetic biology already exist, there are not yet any tools specific to specification of biosecurity features. This working group concluded that it would be useful to have such a software tool created, and discussed features that would be ideal to incorporate in such a tool. We thought that integrating security considerations into desirable system properties such as 'biocontainment' features would be desirable. We also thought it would be an opportunity to specify whether or not DNA sequences of known threat status should be incorporated into designs, or not, and create a direct link to downstream design tools.

The creation of such tools can also lead towards creation of design standards with respect to biosecurity. While our working group decided that biosecurity specification standards were a good idea, it is less clear how they would be enforced. For other specification topics in the field, complying with a specification standard is voluntary best practice. In some limited cases, like within the iGEM community, a form of project specifications with security considerations are submitted to the central organization for approval before specific designs are made, but this is not a scalable practice. In principle, a decision tree could be deduced to help an engineer

\footnotetext{
${ }^{16}$ Appleton, Evan, et al. "Design automation in synthetic biology." Cold Spring Harbor perspectives in biology 9.4 (2017): a023978.
} 
determine if a specification should move forward or not, but most likely in the short term, complying with standards would have to remain a voluntary best practice.

\section{Design Tools}

After the specification step of design comes the selection of specific components to satisfy the specification. This is still most often done manually by a user in various software interfaces, but can also be done automatically with design tools. The output of this design step in a synthetic biology context is a complete DNA sequence. While current genetic design software generally does not screen the DNA sequences at this step for hazardous fragments, there is an opportunity in that step to perform an in silico screen in this design step for potentially hazardous sequences.

While the DNA synthesis and assembly step is a clear place to look for matches to known threats, design tools could be an ideal environment to perform modelling and analysis for potentially less obvious threat. In principle, whole-cell modelling could be developed to determine if over-expression of certain agents will disable cell metabolism via Flux Balance Analysis. ${ }^{17}$ Models could also be developed to predict if a specific protein resembles a hazardous agent or if a viral agent could pose risk to a specific model system or cell type.

These types of modelling approaches with a complete DNA sequence could be extremely valuable at mitigating biosecurity risk before synthesis, but it is known that very few groups are capable of making such models at this time. And even then, it's hard to validate these models completely to the point that these design tools can be used reliably. Although there have been some powerful design tools recently published with highly successful design automation functionality for genetic logic circuits, ${ }^{18}$ building tools for biosecurity threats would be a bit more abstract and require knowledge of the environment and potentially a community of cells on top of whole-cell modelling. This could be simplified for in vitro cell-free systems, but it is general knowledge that one cannot directly apply knowledge obtained in in vitro systems to in vivo systems.

\section{Selecting Chassis}

Once a final DNA sequence for a design is determined, it must be determined which 'chassis' (i.e. organism/model system) the DNA will be used in. Generally this information is already included in the DNA design, but when you consider the environment in which the DNA is introduced to a cell, additional considerations become relevant (especially biosecurity considerations). Furthermore, traditionally

\footnotetext{
${ }^{17}$ Orth, Jeffrey D., Ines Thiele, and Bernhard Ø. Palsson. "What is flux balance analysis?." Nature biotechnology 28.3 (2010): 245-248.

${ }^{18}$ Nielsen, Alec AK, et al. “Genetic circuit design automation.” Science 352.6281 (2016).
} 
some complete organisms have been designated as dangerous agents (i.e. Yersinia pestis) - while the organism has pathogenicity as a whole, a vast majority of the genes in these cells is harmless to humans. It would be valuable to create design tools that also consider the functionality of the chassis the DNA is implanted into for example is it possible to add DNA to chassis that either makes a previously harmless chassis harmful or a previously harmful chassis harmless?

The simplest chassis is one that only uses cellular components, but no complete cell (i.e. 'cell-free systems'). The use of these chassis can simplify the analysis of whether an agent is harmful, but if this DNA were to get inadvertently to a living organism, it would be hard to know if it could become harmful or not. The most complicated chassis use case is a future unnatural, engineered organism or an organism that is rarely used and poorly understood in the literature. In this case, it would be near impossible to say with certainty if a DNA sequence is harmful in these contexts. This can get further convoluted if one must determine which organism or type of cell is harmed, as not all biological threats to humans are direct threats to human cells (i.e. threats to agriculture). In summary, there is ripe opportunity to develop tools that consider the organisms DNA is used in when determining DNA threat status.

\section{Tools to Enhance Tracking of Users and Research}

Finally, in addition to the many purely technical opportunities that exist for biosecurity development in the near future, there is also the opportunity to track activities at the design phase. Right now, all DNA threats are typically caught at the Build step, but if a user logs all of their design thinking, there is greater ability to warn a user of potential DNA threats before they start physical construction of the DNA. If their design was linked to databases where threat information can be automatically queried, projects that accidently use hazardous DNA components can be mitigated earlier. In the later future, if these types of tracking and logging were done at the design level, additional high-level adaptive management of new threat information could be incorporated seamlessly.

\subsubsection{Build}

After the design process, the next step of a biological engineering process is to strategize the build step. In this case, our construction material is DNA. As discussed in this chapter, there are already some existing biosecurity frameworks that relate to DNA synthesis and assembly and some gaps. In this section, we discuss some detailed near-term opportunities for technology development and technical guidelines in the Build phase.

Over the past 10 years, there has been huge technological advancement in the DNA synthesis field and has caused a shift in DNA synthesis versus DNA 
assembly. ${ }^{19}$ In the past, a majority of DNA building was done in labs via PCR amplification and DNA assembly. This was necessary for building large constructs, as it was only economical to synthesize short fragments of DNA called DNA oligonucleotides (i.e. 'oligos'). However, because technology DNA synthesis of large fragments has become so much cheaper, it is now often more economical to simply outsource synthesis of most fragments and only assemble these large fragments in the last step, as opposed to relying primarily on traditional molecular cloning. As a consequence, most imminent opportunities in this space fall under the purview of DNA synthesis as opposed to DNA assembly. The working group identified a number of concrete avenues for strengthening current screening procedures and practices.

\section{Who Should Be Screening Synthesis Production in the Future?}

One general gap identified in the prior section was a lack of consensus on who ideally should be screening synthesis production. While the current system has the DNA producer (i.e. synthesis company, bench biologist, etc.) self-regulating using guidelines, as DNA synthesis becomes a more and more accessible technology, this might not remain the case. The two primary alternatives to self-regulation would be having licensed companies that provide DNA screening as a paid service or having government agencies commit resources to perform this service. Each of these alternatives has pros and cons, but both approaches generally require a centralized screening tool.

The benefit of having governments in charge of screening DNA orders is that they have a direct link to regulatory structure and no direct incentive for profit. It would also be advantageous from a centralization perspective - it could have one screening tool and one database and would not require verification that multiple screening tools and databases are screening correctly. Furthermore, a government is able to control exports of physical items (i.e. DNA). This could be a good mechanism for ensuring that hazardous DNA is not produced and exported, with legal punishment for violators. In addition, the government has access to additional information via state intelligence programs to perform customer screening (i.e. a government would have existing lists of individuals and organizations considered dangerous that it would not allow synthetic DNA to be delivered to). If done in an ideal way with neutrality towards the DNA synthesis producers and consumers, the government screening option could be a good solution. Unfortunately, in reality it might not be that simple. Currently this industry functions void of almost any regulation - adding regulation is always a messy process and each government has different attitudes towards business and science. Furthermore, a rogue state with

\footnotetext{
${ }^{19}$ Hughes, Randall A., and Andrew D. Ellington. "Synthetic DNA synthesis and assembly: putting the synthetic in synthetic biology." Cold Spring Harbor perspectives in biology 9.1 (2017): a023812.
} 
control over the DNA synthesis industry could become a more general existential threat. $^{20}$

Another alternative is having licensed companies providing screening as a service to the DNA producer. While one such company already exists, ${ }^{21}$ more could exist in the future. Like with the government solution, this approach uses a centralized tool and database to perform screening, but is managed by a company, which has different incentives and concerns. One advantage of this solution is that it is consistent, but also is not directly tied to regulations, which allows the synthesis producers to operate with more freedom. The main complexity of this solution lies in intellectual property concerns - namely that companies have interest in not divulging their IP to other companies under the concern that the company that is screening could in principle use sequence information from the screen for economic gain. This solution requires a high degree of trust between the companies producing DNA and those screening it. This proposed solution raises all sorts of liability issues that must be negotiated between all participating parties and could be more complex than a government-based solution.

\section{A Stratified White List Approach for DNA Synthesis Production}

After a clear decision is made on the future of which parties will be performing DNA synthesis screening, a general strategy on which DNA should and should not be allowed for synthesis must be formulated. Under today's current guidelines, screeners use a Black List approach - all sequences are allowed except those that closely match DNA sequences designated as potentially hazardous. When DNA consumers make requests that are on the Black List, their orders are flagged and the DNA producer will follow up with the consumer to verify whether or not they should get the DNA. While this solution works well for sequences of known threat (i.e. fragments of the Smallpox genome), it is not able to handle new threats or threats which are not currently deemed hazardous enough to make the Black List. The result is that there is likely a large volume of sequences produced and distributed today that have some sort of threat potential. The Black List approach works well if the list remains static, but we know that in the synthetic biology space, this is not a realistic expectation.

The reverse of the Black List approach is a White List approach - a White List contains a large library (or generic definition) of sequences that cause no reason for concern. In a White List-centric approach, only things that give hits on the White List are allowed and anything not on it is not allowed. The primary difference between White- and Black-List approaches is how the 'grey' area is approached. In a Black List paradigm, things in the grey area are allowed and resources are spent to

\footnotetext{
${ }^{20}$ Trump BD, et al. Building biosecurity for synthetic biology. Molecular Systems Biology. 2020.

${ }^{21} \mathrm{https}: / / \mathrm{www}$. battelle.org/inb/threatseq
} 
confirm that they aren't technically on the Black List. In a White List paradigm, things that are in the grey area are generally not allowed.

This working group proposed the idea of a 'Stratified White List' approach. In this framework, it is essentially a White List approach with exceptions for highly trusted partners. These highly trusted partners could be institutions with clear approval to work with specific hazardous sequences - depending on the research being performed, there would be different categories of White Lists. The proposed category breakdown of Stratified White Lists could have the following types of breakdowns:

1. Basic molecular biology labs with institutional approval to do work in BL1 (or equivalent) with no declared intention of working on sequences that might pose threat

2. Labs with permission to work on one specific agent or set of agents with established threat status

3. Labs with permission to work on a broad set of agents with known threat status

By default, all customers (new and existing) would be automatically placed in the CATEGORY 1 provided that they have proof that they are working in an established institution (i.e. not a private address with no specific permission to work with DNA) and would then need to pass some certifications to move into CATEGORY 2 or CATEGORY 3. CATEGORY 1 would include labs at academic and industrial institutions and DIY community labs. This certification process would need to be of minimal burden to the customer, but make it clear that the customer has institutional approval to work with certain types of agents to be approved for higher categories. This process could potentially be tied to IGSC or managed in some part by a similar organization.

In general, the Stratified White List system would cover most examples of DNA to be produced, but there are a couple important edge cases that would require more thinking. First, certain mammalian genes (i.e. insulin) could be overexpressed in certain situations that make a gene on the White List cause harm to human cells. Second, this approach still does not solve the problem of sequences that are requested that match no known DNA sequence in the screening database.

While the Stratified White List approach gives a clear tiered system, one issue that could arise is how to deal with the use of middlemen or intermediary institutions giving access of higher-tiered DNA to those at a lower tier. For example, instead of a bench scientist ordering directly from a DNA producer, they might regularly order through a local supplier. Or, for example, a user at a high category clearance giving inactivated forms of agents to lower-category parties under the premise that the second party will not mutate the agent back into active form. To solve this problem, we would recommend an end-user certificate to validate that the party physically using the DNA is on the right category White List. While this proposed system does not completely solve this 'middleman problem', this problem is also unsolved in the current Black List approach. 


\section{Functional Equivalence of Sequences}

The current foundation for determining if a sequence should be built or not is founded on lists of known sequences of harm. However, it is broadly acknowledged that there is a much larger list of sequences that may be threats that are not currently on these lists. This is currently unaddressed in any screening framework, but there has been discussion of methods to assign functional equivalency of sequences - the task of determining if a sequence is 'similar enough' to a known threat to cause pause before DNA synthesis.

Discussions as recently as $2008^{22}$ had deemed this scientific pursuit too challenging of a problem to seriously consider. At that time, it was thought that a nucleotide sequence similarity percentage of $80 \%$ could be useful to identify sequences of potential threat. This sequence matching approach had many problems, namely because sequence identity isn't necessarily a good predictor of function, and was subsequently abandoned. However, in recent years, there have been huge advances made in machine learning in biology and an explosion of DNA production for genetics research. Moreover, at the time, a lot of sequence databases where new and therefore were sparse and contained errors. Now that vast, accurate databases of sequences exist and machine learning in biology has gotten off to a strong start, perhaps it is time to revisit the idea of building tools to predict functional equivalency not based solely on nucleotide sequence.

\section{DNA Assembly and Smaller DNA Synthesis Providers}

As discussed, a large shift in recent years has gone towards de novo DNA synthesis over traditional DNA amplification and assembly. However, DNA assembly of small fragments is still performed widely in the community too. While this DNA assembly is more time consuming and sometimes more expensive than DNA synthesis, it creates some problematic edge cases for the existing screening framework. First, since the current screening guidance only focuses on fragments of size $\geq 200 \mathrm{bp}$, one could order a bunch of small fragments of a hazardous agent and assemble them in a lab without being detected. Second, if a user already has access to some fragments of hazardous DNA, they can order oligonucleotides to mutate and assemble full-length agents. A near-term opportunity in this space is to build software tools that account for DNA assembly. Software could also be developed such that a DNA purchaser account could be flagged if they order a large set of small fragments that partially match a known dangerous agent or if an account suddenly logs 'unusual' ordering activity as is done sometimes with ATM withdrawals at financial institutions.

\footnotetext{
${ }^{22}$ National Research Council. 2010. Sequence-Based Classification of Select Agents: A Brighter Line. Washington, DC: The National Academies Press. https://doi.org/10.17226/12970
} 
The DNA assembly problem is an important area to address since many types of parties still do this routinely. Organism design companies, automated platforms, cloud labs, CROs, guide RNA service providers, and other service providers regularly produce small DNA fragments in house. This issue will become even more pressing for bench-top DNA synthesizers.

\section{Attribution and Tracing}

Finally, while attribution tools have gotten off to a strong start, there are additional angles that might be factored into these tools to include lab-specific optimizations of codon optimization and synthetic biological parts usage patterns. The synthetic biology community often uses different codon optimization schemes for their parts and often re-uses combinations of characterized parts to build complex genetic circuits, so these additional dimensions could strongly aid existing attribution efforts.

In recent years, there has also been a widespread adoption of 'DNA barcoding' techniques for many areas of biotechnology and there could also be opportunities to institute a DNA barcoding system for DNA synthesis in certain capacities. This would require standardization and a consensus of how to do the barcoding, but it could be a useful way to program attribution into the DNA synthesis workflow.

\subsubsection{Test}

The final part of the Design-Build-Test cycle is Test - methods for obtaining and analysing data. While the Design and Build phases predict or assume a certain degree of functionality of an agent, in the Test phase, these qualities are scientifically determined. In the context of biosecurity, this is where the actual threat capability of any given agent is determined. In this situation, a lot of the technological focus is under what setting (both physical and biological) the testing of potential bio-threats is done, since it is dangerous to test potentially hazardous agents in an open, uncontrolled setting. Ergo, most relevant concerns with respect to testing revolve around containment.

\section{Physical Containment}

The first layer of containment is physical containment - where certain types of agents are stored and worked with by scientists. While this is conventionally performed in physically secure labs with different levels of chemical and biological agent clearance, emerging DNA technology has made this problem more complex. Specifically, with the widespread use of synthetic DNA and incorporation into model organisms, do standard decontamination and waste procedures suffice for eliminating biothreats? 
In general, biological waste is either treated with bleach before being poured down the drain or sent for incineration (re-usable containers for research materials are autoclaved at high temperature and pressure). It is assumed that these procedures are broadly effective at containing biological threats, but given the stability of DNA, this generalization should be revisited in near-term research. It is known, for example, that standard sterilization techniques do not fully degrade double-stranded DNA fragments, ${ }^{23}$ leaving whole genes unmodified. While it is not known how much DNA would be needed to create horizontal gene transfer, there is a knowledge gap of ramifications of allowing DNA to escape labs via current sterilization processes. This working group identified the area of measuring levels synthetic DNA in waste collection and the general environment as an area of key opportunity in physical containment. As an extension, another area of imminent technology development would be using new technologies to set up a surveillance network to track when DNA fragments of interest are detected at specific physical locations. Such a surveillance network could ground many of our assumptions on the physical spread of biologics from laboratories.

\section{Biology-Based Containment}

A second layer of containment is biology-based containment. Biology-based methods contain organisms using programmed biological features. The key difference in this containment approach is that it allows engineered organisms in the environment outside of a controlled facility. While this is traditionally avoided, there could be large benefit of using engineered organisms in the environment for applications such as bioremediation where organisms could be used to clean the environment of toxic molecules or pollution.

One early biology-based containment method is the use of antibiotic resistance genes such that only bacteria with that gene could grow on a substrate. Later on, other approaches of biology-based containment were developed including using cell lysates (i.e. cell-free systems) and partial organisms (i.e. lenti-viral packaging) to control biological spread by removing parts of the biology used for replication. In more recent years, technologies such as recoding, ${ }^{24}$ kill-switches, ${ }^{25}$ and gene drives ${ }^{26}$ have been introduced to engineer biocontainment such that organisms can be used in certain field applications without the ability to escape the controlled environment.

\footnotetext{
${ }^{23}$ Yap et al. "Integrity of bacterial genomic DNA after autoclaving: possible implications for horizontal gene transfer and clinical waste management" Journal of Hospital Infection 83 (2013) pp 247-249.

${ }^{24}$ Lajoie, Marc J., et al. "Genomically recoded organisms expand biological functions." science 342.6156 (2013): 357-360.

${ }^{25}$ Stirling, Finn, et al. "Rational design of evolutionarily stable microbial kill switches." Molecular cell 68.4 (2017): 686-697.

${ }^{26}$ Esvelt, Kevin M., et al. "Emerging technology: concerning RNA-guided gene drives for the alteration of wild populations." Elife 3 (2014): e03401.
} 
Kill-switch technology describes programmed mechanisms for a human observer to change the environment where an organism is placed in order to cause the organism to rapidly die. This has been engineered for both temperature and environmental triggers. Additional technology development for fine control of these mechanisms, using genetic logic gates ${ }^{27}$ or an engineered micro-biome ${ }^{28}$ could provide more sophisticated control of containment. Technology development in this area would have high near-term impact for biosecurity, as we think about how to introduce new, impactful biological applications, while taking proper measures to be able to control the spread of engineered organisms if they are not behaving as desired. It could also impact the desirability of biological weapons, should we develop capabilities to accurately confine engineered systems to specific locations.

A second new technology for biocontainment, called 'recoding', is a method for containing engineered agents by requiring them to use an alternate genetic code for survival. This has been done for $E$. coli, where these recoded bacteria, called $r E$. coli, require the addition of extra unnatural amino acids in the environment to survive, and thus cannot grow in environments that do not have an unnatural additive. ${ }^{29}$ There is on-going work in the field to expand this technology into new organisms and at greater scale. Further near-term development in this area will lead to creation of organisms that are safe for use in the environment because they fundamentally cannot survive in natural environments. A key step will be experimentally demonstrating that this is true.

A third new technology, 'gene drives', ${ }^{30}$ has been proposed as a genetic mechanism for control of population genetics. This technology uses engineered inheritance to guarantee the passing of certain genes via sexual reproduction in eukaryotes. The result is that populations could, in principle, be culled or controlled using the gene drive mechanism. The advent of this technology has drawn in large-scale science funding ${ }^{31}$ to determine if this approach is has efficacy on a large scale and develop technologies such as reversible gene drives to correct potential mistakes made. There have also been efforts to limit the spread of gene drives to specific locations. ${ }^{32,33}$ The primary model organism used thus far for gene drives is the mosquito, since suppression of mosquitos in certain regions could be used to supress the

\footnotetext{
${ }^{27}$ Tamsir, Alvin, Jeffrey J. Tabor, and Christopher A. Voigt. "Robust multicellular computing using genetically encoded NOR gates and chemical 'wires'.” Nature 469.7329 (2011): 212-215.

${ }^{28}$ Pacheco, Alan R., Mauricio Moel, and Daniel Segrè. "Costless metabolic secretions as drivers of interspecies interactions in microbial ecosystems." Nature communications 10.1 (2019): 1-12.

${ }^{29}$ Mandell, Daniel J., et al. "Biocontainment of genetically modified organisms by synthetic protein design.” Nature 518.7537 (2015): 55-60.

${ }^{30}$ Esvelt, Kevin M., et al. "Emerging technology: concerning RNA-guided gene drives for the alteration of wild populations." Elife 3 (2014): e03401.

${ }^{31}$ darpa.mil/program/safe-genes

${ }^{32}$ Marshall et al. "Can CRISPR-Based Gene Drive Be Confined in the Wild? A Question for Molecular and Population Biology." ACS Chemical Biology. 13, 2 (2018) pp 424-430.

${ }^{33}$ Sudweeks et al. "Locally Fixed Alleles: A method to localize gene drive to island populations." Sci Rep 9, 15,821 (2019). https://doi.org/10.1038/s41598-019-51994-0
} 
spread of malaria and other diseases. This area is ripe for additional technology development and application to more species if it can prove controllable in current research efforts.

This working group discussed ways in which the existing methods could be used synergistically to create additional layers of biocontainment. For example, one could imagine a situation where artificial dependence on certain conditions and dependence on antibiotics triggers the expression of certain factors in absence of the antibiotic to kill the cells could be a two-component system. This type of containment system could exist between an animal and bacterium where they depend on each other and one dies out in the environment without the other. Other 'xenobiotic' biocontainment examples could be developed to create complex, layered levels of biocontainment in the near future. This type of path forward will require much greater inclusion of ecologists in relevant research areas. Even then, there will be some risk in such projects. Some reversion might be possible by use of kill switches and reversal drives, but it is likely that some changes will be permanent depending not only on the system but also the population size, where it is released, and the fitness of the organism in the environment it is released into.

\section{Horizontal Gene Transfer}

Finally, in hypothetical cases of genes escaping containment, we must consider how to mitigate horizontal gene transfer. In principle, horizontal gene transfer has a certain pace with which new DNA gets introduced to a new bacterium by chance. The new DNA often has only a limited beneficial metabolic function and will for sure not be toxic to the cell. Evolution may change the DNA into genes with a more central role in metabolism or increase expression, otherwise the DNA may be lost again. In a modern global world of today and the invention of many different antibiotic drugs, the selective pressure on the bacteria has never been greater and only the most pathogenic strains can survive. Thus the acquisition of gene cassettes varies greatly.

Bacteria employ a variety of mechanisms to transfer genes horizontally, such as transformation, transduction and conjugation. Natural transformation is a process by which cells take up naked DNA from the environment. It involves multicomponent cell envelope spanning structures, such as type II secretion systems (T2SS), type IV secretion systems (T4SS) and type IV pili. In transduction, DNA is transferred with the help of bacteriophages and conjugation requires physical contact between a donor and a recipient cell via a conjugation pilus, through which genetic material is transferred.

So what is transferred? A broad spectrum of mobile genetic elements, such as plasmids, transposons, bacteriophages or genomic islands are transferred and can be found to account for a large proportion of bacterial genomes as evolution goes on. An example of selective pressure is the acquisition of copper resistance (along with resistance to arsenic and cadmium) - comprising czc/cusABC and copABCD 
systems in the kiwifruit pathogen Pseudomonas syringae pv. Actinidae. ${ }^{34}$ The pathogen infected the first plantation in Australia in 2010, and by 2016, 25\% of all samples taken were resistant to the copper treatment.

With the development of modern molecular biology tools, endless new DNA constructs have been released into nature when biological waste is deliberately or by accident tossed down the drain. Resistance marker genes, plasmids with multihost capabilities, and fusion proteins are a great source of DNA that can be taken up by other bacteria and which will make them even more pathogenic to human health than before.

\subsubsection{Economic Drivers}

While we have discussed here many areas of imminent technology development that could significantly bolster biosecurity practices, we must not forget the underlying economic incentives of DNA production, since economic drivers important to realising the potential of synthetic biology run counter to comprehensive biosecurity governance. Commercial applications inherently want to maximise profit and minimise overheads. Creating and implementing measures to prevent deliberate misuse add cost. This is a notable disincentive for large parts of the synthetic biology community to engage with biosecurity. Therefore, it is particularly important to streamline the financial and resource implications of biosecurity measures.

Furthermore, given its intrinsically interdisciplinary nature, many members of the synthetic biology community come from disciplines outside biology and biotechnology. As a result, they may not have been exposed to, or have a background in, biosafety or biosecurity. It is therefore important that biosecurity measures are accessible, supported by appropriate tools and resources, and adequately promoted among members of the community.

\subsubsection{Detection of Biological Threats}

Thus far, we have discussed numerous ways in which biological threats either are currently being mitigated or can be mitigated with technology development in the near future. However, there are also numerous opportunities to increase capability in the threat detection domain. In this domain, we assume that a biological threat has already been physically created in the environment and the question focuses on technology to detect it. In this context, we consider our ability to establish surveillance methods and rapidly diagnose biological threats.

\footnotetext{
${ }^{34}$ Colombi, Elena, et al. "Evolution of copper resistance in the kiwifruit pathogen $\mathrm{P}$ seudomonas syringae pv. actinidiae through acquisition of integrative conjugative elements and plasmids." Environmental microbiology 19.2 (2017): 819-832.
} 


\subsubsection{Diagnostics}

Current methods for diagnostics of hazardous agents can be summarized as a collection of peptide sequencing, antibody-based diagnostics via ELISA or ImmunoPCR, and genome sequencing technologies. Generally speaking, peptide sequencing is most useful for protein threats like toxins, antibody screening is most often used for viral infections, and genome sequencing can be performed for both viruses and bacteria.

Of these technologies, the most rapidly evolving is genome sequencing. While some companies have made great progress on portable whole-genome sequencing, ${ }^{35}$ there are still significant issues with the limit of DNA detection. Often there is not enough genetic material acquired in the field to make confident identifications of species and if the organism is modified, it makes that conclusion even more difficult. Some new microfluidic devices have aided this problem, but there is still a lot of room for improvement. Additional technological progress in microfluidic device development is a key area of opportunity to improve these DNA-based diagnostics. Another issue for these diagnostic devices is the comparison to reliable, nonredundant sequence databases. Historically, as large sequence databases have been built, a fair amount of inaccurate data and erroneous meta-data has been entered and so another opportunity to improve the diagnostic functionality is to clean up these databases to the point that they can be much more useful for immediate comparison with diagnostic devices.

One key area of diagnostic development for a variety of agents is cell-free systems. Cell free systems have been used for many years and are routinely produced by individual laboratories following their own recipe. Today the technology has advanced and the understanding of the important factors to make reproducible kits has enabled it to be used outside the lab. ${ }^{36}$

Toehold switches were developed in 2014 and utilized the preferential binding of DNA into a secondary structure if no target was present and it would unfold and bind to the target if it was present. A reporter gene would be activated upon unfolding and a signal could be detected. The technique can detect nanomolar and low micromolar concentrations due to the absent amplification step of target. It will generate signal detection in as little as $20 \mathrm{~min}$ and the maximum ON/OFF ratios ranged between 10- and 140-fold. Careful optimization of target region is needed to ensure maximum signal. An important advantage of paper-based distribution of synthetic gene networks is their potential for low cost $(4-65 \not /$ sensor) and relative ease to manufacture. ${ }^{37}$

\footnotetext{
${ }^{35}$ https://nanoporetech.com/

${ }^{36}$ Takahashi, M.K., Tan, X., Dy, A.J. et al. A low-cost paper-based synthetic biology platform for analyzing gut microbiota and host biomarkers. Nat Commun 9, 3347 (2018). https://doi. org/10.1038/s41467-018-05864-4

${ }^{37}$ Pardee, Keith, et al. "Rapid, low-cost detection of Zika virus using programmable biomolecular components." Cell 165.5 (2016): 1255-1266.
} 
Developing the technique further, by using Cas9 and an isothermal RNA amplification step, the detection limit improved to low femtomolar range and had a single-base resolution discriminating between American and African Zika type viruses. Other variants of the technology (Sherlock) use an isothermal RNA amplification step to get low attomolar sensitivity by using a different Cas 13 protein. This protein will, via the CRISPR methodology, find its target and cleave it. ${ }^{38}$ Due to a build in collateral cleavage feature, the Cas 13 protein will next degrade any mRNA it may find. Thus the provided fluorochrome - quencher reporter mRNA molecule will be degraded and a signal can be measured using a fluorescent reader. Yet again, other systems using cas 12 can target DNA in the same way as mentioned above ${ }^{39}$ or by using a CRISPR-Cas9-triggered nicking endonuclease-mediated Strand Displacement Amplification method named CRISDA. ${ }^{40}$

A technology to be fully developed in the future is the biological transistor. It is the detection of an unamplified target gene via CRISPR-Cas9 immobilized on a graphene field-effect transistor. An electrical signal is generated if CRISPR detects its target and thus can positively identify a biological agent on the DNA level within $15 \min ^{41}$

With the invention of the methods mentioned here, a field deployable paper stick technology will be able to tell if a dangerous pathogen is present in a fast and reliable way. It is a huge step in the direction of being able to detect a biological attack on site, but still laborious work is still needed to extract nucleic acids from each sample and ID RNA is present, RNases are to be avoided at all cost to get reliable results.

\subsubsection{Surveillance}

Finally, as diagnostic tests are developed to be faster and more accurate, we can start to form systematic surveillance protocols. This can range from detection of immediate human health pathogens to analysis of field micro-biomes, detection of fungi and decomposers, and agricultural pathogens. While these other types of threats are currently too low priority to focus diagnostic efforts on, in the big picture, these areas really matter. In agriculture, there are already systems for tracking and regulating pedigree of lines of animals and plants, but as the technology for diagnostic DNA tests improves, it would be reasonable to develop the areas of

\footnotetext{
${ }^{38}$ Gootenberg, Jonathan S., et al. "Multiplexed and portable nucleic acid detection platform with Cas13, Cas12a, and Csm6." Science 360.6387 (2018): 439-444.

${ }^{39}$ Chen, Janice S., et al. "CRISPR-Cas12a target binding unleashes indiscriminate single-stranded DNase activity.” Science 360.6387 (2018): 436-439.

${ }^{40}$ Zhou, Wenhua, et al. "A CRISPR-Cas9-triggered strand displacement amplification method for ultrasensitive DNA detection.” Nature communications 9.1 (2018): 1-11.

${ }^{41}$ Hajian, Reza, et al. "Detection of unamplified target genes via CRISPR-Cas9 immobilized on a graphene field-effect transistor." Nature biomedical engineering 3.6 (2019): 427-437.
} 
genetic surveillance of agriculture since it is a high-impact area of human wellbeing that is not directly human health focused.

\subsubsection{Threat Response and Countermeasures}

Biological countermeasures are typically biologics and small molecules used to detect, prevent, or treat biological and chemical insults. Biologics are composed of vaccines and antibodies. Vaccine development, while slow and laborious, is effective at producing acquired immunity and protection to a broad range of known diseases and weaponized agents. Recently, large-scale mining of human immune repertoires for antigen binders has been propelled by technological advances such as next generation sequencing (NGS) and given rise to the field of system-immunology. Coupled with bioinformatics analysis, we have gained significant insight into the diversity of antigen binders and the polarization of repertoires in response to challenge. Subsequently, it is now possible to mine these repertoires for protective monoclonal antibodies and deliver effective countermeasures. However, extant antibody discovery platforms suffer from a multitude of disadvantages that impede high-throughput repertoire interrogation and antibody discovery.

There are several large existing programs to develop medical countermeasures (MCMs) to new and existing biothreats such as p3, PRISM, and US AID, but these programs are beyond the scope of near-term biosecurity efforts to improve. While new synthetic biology tools will certainly lead to decreased development time to countermeasure delivery compared to traditional approaches, it is still relatively new technology and will take some time to be integrated into these large existing efforts to develop countermeasures.

One topic discussed at some length by this working group was how information on MCMs should be disseminated to the general public. Specifically, we discussed the idea of putting the latest technical information on new threats online. For example, each year there is a seasonal strain of influenza that circulates and vaccines are routinely developed to combat the new strain. To do this, the new viral strain is sequenced and a new MCM is created. Should these new viral sequences and information on countermeasures be publicly available information?

Historically this type of information has been available only to those actively working in the space. New sequence information is kept on a non-public database and companies that develop yearly MCMs for influenza get the physical strain in exchange for donating some vaccine free of charge for use in resource limited settings. However as they can access the data with the virus sequence, they do not have to ask for the strains anymore - the can simply synthesize the new strains themselves, make the vaccines from that source without the obligation to make the MCMs more generally accessible. Thus, the system relies on the good will of the companies to share benefits from their work. 
Furthermore, we have seen a much more open approach in response to the global COVID-19 pandemic - the DNA sequence for this threat ${ }^{42}$ and subsequent diagnostic and countermeasure development has been rapidly published and made publicly available. ${ }^{43}$ This has led to rapid development of novel diagnostics and MCMs by a large swathe of companies (including some synthetic biology-based companies), giving tremendous opportunity for the biotechnology industry. But, the cons of this very open approach will take time to play out, as some risk has been taken by disseminating so much of this information in a short period of time to mitigate a global crisis. Only after the dust settles with the on-going COVID 19-pandemic, will we be able to see whether or not there are clear negative consequences of this open approach.

\subsection{Long-Term Opportunities for Technology Development}

The discussion in this chapter has thus far discussed the existing technical biosecurity framework and various areas for near-term improvement. Here, we have some additional discussion of long-term opportunities for improvement that are still too nebulous to attempt to mitigate with cutting edge technologies in the short term future.

\subsubsection{Implications for Adaptive Risk Management Framework}

Several aspects of technologies and products derived from synthetic biology create a need for an adaptive approach for risk assessment. ${ }^{44}$ Synthetic biology approaches are foundational, being applied to many different problems/areas and accessible to a wide range of people. Because of this, there is great uncertainty about what type of technologies might develop from synthetic biology. At the same time, new products and capabilities are being developed at a rapid pace. As a result, it is difficult to anticipate the potential threats posed by synthetic biology using a prescribed or static approach for assessing risks and bio-threats.

\footnotetext{
${ }^{42}$ Andersen, Kristian G., et al. "The proximal origin of SARS-CoV-2." Nature medicine 26.4 (2020): 450-452.

${ }^{43}$ Thao, Tran Thi Nhu, et al. "Rapid reconstruction of SARS-CoV-2 using a synthetic genomics platform." BioRxiv (2020).

${ }^{44}$ Oye, K. "Proactive and adaptive governance of emerging risks.

The case of DNA synthesis and synthetic biology." Public Sector Governance of Emerging Risks, International Risk Governance Council (2012) https://irgc.org/wp-content/uploads/2018/09/ FINAL_Synthetic-Biology-case_K-Oye_2013.pdf
} 
We were able to identify desirable features of an adaptive risk management framework. First, an adaptive risk management would need to use widespread monitoring and data collection practices. This would likely include large-scale measurement of known hazardous DNA and engineered DNA. Secondly, there would need to be a systematic process for horizon scanning to identify new things that realistically might need to be measured. It is not feasible to have a single static list of concerns in a world where biotechnology is constantly evolving. Such a system could look a little like monitoring data from subway filters ${ }^{45}$ with indicators of background levels of specific agents. First such backgrounds would need to be clearly established and there would have to be a mechanism to flag the presence of new, unknown particles.

\subsubsection{Imagining the Far Future}

Finally, the group carried out a future-casting exercise where participants imagined what the world might look in the long-term future (25-50 years). They considered the role synthetic biology (and its products) could play in that world. Such a future could be positive or it could be negative. This exercise involved scenario development, group work to identify specific biosecurity implications, and then back-casting to identify key technological developments that might be important for shaping the future.

Possible future applications of synthetic biology identified included:

- Enhanced diagnostics, such as those based on a comprehensive understanding of life processes

- Cheaper, faster and improved medicines (including at point-of-care), such as personalised medicine, smart wound healing materials, antibiotics, vaccines, other biologics (including to balance the metabolism)

- Human enhancement, such as brain/visor interfaces, augmented limbs, synthetic organelles to correct natural deficiencies (such as delivering insulin for diabetics) or communication with animals

- Microbiomes engineered to be self-balancing, offer improvements to performance, health or a more robust immune system, or more efficient in processing and absorbing nutrients from food

- Adjusted plants, resistant to challenges from climate change and food shortages resulting from expanding human populations

- On going, near-real-time remote monitoring of health and the environment

- Chemically-induced exercise through the diet

- Enhanced food, including with a long shelf life

\footnotetext{
${ }^{45}$ Grass, David S., et al. "Airborne particulate metals in the New York City subway: a pilot study to assess the potential for health impacts." Environmental research 110.1 (2010): 1-11.
} 
- Improvements to the transport infrastructure, such as self-repairing or better road surfaces

- Changes to energy use, such as by increasing use of bio-lights

- Greater control over the environment, such as increasing biological diversity, through bioremediation, systems for reclaiming or making use of waste, controlling or removing pests and disease vectors, or perhaps weather control

- Cyber-genetics and enhanced immune surveillance

- Enhanced materials, such as materials that adapt to the environment, alternatives to, or new ways to recover rare earth metals

- Novel production capacities, such as directly from carbon and water, possibly via distributed production, to make things like food or high-value chemicals

- Point-of-use (distributed) manufacturing, such as through the use of wellcharacterized and reliable chassis organisms.

The group then split into three sub-groups based on common components of their visions of the future. The sub-groups then discussed relevant biosecurity issues, focusing on ways that a lack of, or adequate, biosecurity could have a notable impact delivering a desirable future. Intervention points identified included:

- The impact of business models in shaping integration of biosecurity and the importance of engaging the private sector on biosecurity early in technology development

- The importance of improved risk assessment and management of biosecurity risks to enable ecological or environmental uses

- Adaptive, flexible, proactive, and cooperative approaches to governance

- Involvement of publics and industry in biosecurity governance and decision-making

- Perception issues around military funding of synthetic biology

- Potential for subversion of neuro-electric interfaces, the importance of encryption, and the potential for cyber attacks to have a biological impact and to hack biological components to have a digital effect

- The possibility of biosecurity concerns to unjustifiably restricting progress in key fields (opportunity costs)

- The value of developing new capabilities to carry out applied risk assessment between the bench and field trials (pilot scale), such as through the use of isolated environments that can provide a baseline for studies

In the same small groups, participants then considered which technical interventions could help address the biosecurity issues identified. These included:

- Rapid dynamic adaptive approach, surveillance system for environment

- Technology to identify the origin of a molecule or tag DNA bases (e.g. fertilizers have to be tagged - > biological tags like isotopes)

- 'Biological encryption' to counter bio-hacking of neurons 
In summary, the working group came up with a wide variety of potential future technologies and their related biosecurity risks. While most of the future technologies indeed had at least one clear biosecurity risk, the group had a mostly positive outlook on the future of synthetic biology and technical biosecurity.

Open Access This chapter is licensed under the terms of the Creative Commons Attribution 4.0 International License (http://creativecommons.org/licenses/by/4.0/), which permits use, sharing, adaptation, distribution and reproduction in any medium or format, as long as you give appropriate credit to the original author(s) and the source, provide a link to the Creative Commons license and indicate if changes were made.

The images or other third party material in this chapter are included in the chapter's Creative Commons license, unless indicated otherwise in a credit line to the material. If material is not included in the chapter's Creative Commons license and your intended use is not permitted by statutory regulation or exceeds the permitted use, you will need to obtain permission directly from the copyright holder. 